\title{
Calculating the Borehole Geometry Produced by Helical Drilling with Ultrashort Laser Pulses
}

\author{
Alexander Kroschel ${ }^{1,2}$, Andreas Michalowski ${ }^{1}$, Franziska Bauer ${ }^{1}$, and Thomas Graf ${ }^{3}$ \\ ${ }^{1}$ Robert Bosch GmbH, Zentrum für Forschung und Vorausentwicklung, \\ Robert-Bosch-Campus 1, 71272 Renningen, Germany \\ E-mail: alexander.kroschel@de.bosch.com \\ ${ }^{2}$ Universität Stuttgart, Graduate School of Excellence advanced Manufacturing Engineering \\ (GSaME), Nobelstraße 12, 70569 Stuttgart, Germany \\ ${ }^{3}$ Universität Stuttgart, Institut für Strahlwerkzeuge (IFSW), Pfaffenwaldring 43, \\ 70569 Stuttgart, Germany
}

\begin{abstract}
Laser drilling is a processing technology applicable for creating holes in various materials. By combining an ultrashort pulsed laser with a helical drilling optics, it is possible to produce high-quality holes with sharp, burr-free edges in a large variety of borehole geometries. To master this highly flexible process which is determined by a multitude of variables, a mathematical description of the problem is advantageous. A simulation model is presented for calculating the final borehole geometry for a given set of laser, process, and material parameters. The model was validated by comparison to boreholes drilled into four different materials: stainless steel, copper, silicon, and aluminum oxide ceramic. By inserting literature values for the refractive index and the ablation threshold fluence, a good agreement between model and experiment can be achieved for holes of different shape and size. DOI: $10.2961 / j l m n .2018 .03 .0020$

Keywords: ablation threshold, helical drilling, laser materials processing, simulation, ultrafast lasers
\end{abstract}

\section{Introduction}

Laser drilling is a manufacturing technology for producing holes, which is used in a wide range of industrial products. There are several process strategies, differing in productivity and hole quality [1]: The highest productivity is achieved with single-pulse drilling and long pulse durations $(\mu \mathrm{s})$; however, here, material removal is dominated by melt ejection which leads to a reduced quality. Percussion drilling using short pulses (ns) offers an increased precision by the reduction of fluence and therefore less burr and melt deposition, but also far more pulses are needed. A further reduction of the pulse duration to ultrashort laser pulses (ps, fs) eliminates melt and burr, as material removal is dominated by vaporization. The high geometric precision is taken advantage of by applying an adequate drilling strategy to modify the borehole form [2].

For helical drilling, one of those process strategies, commercially available optics [1,3-7] are used which offer the possibility to move the laser beam in a rotary movement with three degrees of freedom: a rotation frequency, a helical radius, and an inclination toward or away from the axis of rotation. Because of its advantages in hole quality and geometric freedom, helical drilling with ultrashort laser pulses offers the potential to complement or replace mechanical or electro-erosive drilling steps by laser micro drilling. An exemplary application for this technology is the processing of spray holes in injector nozzles [2,3].

In a previous work [7], we presented a model predicting the final borehole geometry produced by helical drilling with ultrashort laser pulses. As explained in Sec. 2, the expansion of the borehole is assumed to stop when the absorbed fluence falls below the ablation threshold fluence. Therefore, the cross-section of the borehole geometry in a steady end state is assumed to equal a line of constant absorbed fluence.

As it is possible to drill into almost any available material using ultrashort laser pulses, the model is validated for four different materials in this paper by comparing simulated and real boreholes. These materials include industrially relevant metals (stainless steel and copper) and non-metals (silicon and aluminum oxide ceramic (alumina)).

As input parameters for the simulation, the focusing conditions of the laser beam, set parameters of the helical drilling process, and literature values for the material properties were used.

\section{Model for calculating the final borehole geometry}

In the laser drilling process, the borehole evolves and expands as long as the absorbed laser fluence is higher than the ablation threshold. The expansion stops in the points where the absorbed fluence falls below the threshold value. Therefore, the isophote (surface of constant absorbed fluence) defined by the ablation threshold fluence can be assumed to equal the steady end state of a borehole produced by an infinitesimally long laser drilling process where all beam, material, and process parameters are constant over time.

While in the actual drilling process an evolution of the borehole geometry takes place until the final hole shape is reached, in the static model, only the steady end state of the hole geometry is considered. Hence, it is assumed that there is no influence of the temporal borehole evolution on the final shape (e.g. bifurcations, reported in Ref. [8]).

For all points on the surface of constant absorbed fluence, it holds

$$
(1-R) \cdot \cos \theta \cdot F_{0}=\tilde{F}_{t h}=\text { const., }
$$


where $R$ denotes the local reflectivity, $\theta$ the relative angle between the incident ray and the local surface normal, $F_{0}$ the local laser fluence, and $\tilde{F}_{t h}$ the constant ablation threshold fluence (particularly referring here to the maximum value of the fluence that is penetrating the surface which does not lead to any ablation).

Assuming an ideal Gaussian fluence distribution, the laser-drilled borehole is rotationally symmetric, meaning that for the calculation, only the cross-section has to be considered, resulting in a two-dimensional problem. Therefore, the line of constant absorbed fluence is computed and compared to the cross-section of a borehole.

In general, it holds $(1-R)=A+T$ with the absorptivity $A$ and transmissivity $T$. Due to the short optical penetration depth, $T \approx 0$ for metals. For dielectrics, however, the penetration depth is much larger, in some circumstances leading to $T>0$. As previously discussed, the simulation result for the final borehole geometry is a surface of constant absorbed fluence. Here, we define that the absorbed fluence includes all radiation penetrating this surface. In the following validation experiments, materials with strong absorption, and thus $T \approx 0$, are used. Therefore all radiation is assumed to be absorbed at the surface. Further research is needed whether this holds true for the ceramic.

The local reflectivity $R$ is described by the Fresnel equations, incorporating the complex refractive index of the material $\bar{n}=n+i k$, where $n$ is the refractive index, and $k$ is the extinction coefficient with $k>0$ for absorbing materials.

For the local laser fluence $F_{0}$, a Gaussian fluence distribution is assumed with the laser beam parameters pulse energy $Q$, waist radius $w_{0}$, Rayleigh length $l_{R}$, and defocusing length $s$.

Additionally, the helical drilling parameters, particularly the helical radius $r_{\text {helical }}$ and the angle of inclination $\beta$, are included in the calculation of the spatial distribution of the incident fluence. As the model is reduced to two dimensions and its purpose is to calculate the final borehole geometry, the rotation frequency is irrelevant.

In the following sections, this model is used to predict borehole geometries based on the experimentally used drilling parameters. The simulation results are compared to real borehole cross-sections.

\section{Experimental procedure}

For the validation of the model, boreholes have been drilled into four different materials. A commercial helical drilling optics was used for the experiments (GL.Trepan included in a GL.Compact machine, both by GFH GmbH, Germany). The setup incorporates the laser source TruMicro 5070 Femto Edition (Trumpf GmbH + Co. KG, Germany).

The general processing parameters used can be found in Table 1 . The focal diameter of the Gaussian-shaped fluence distribution has been determined after the method described in [9]. Using the same set of drilling parameters for each material, several holes have been produced respectively with different $d_{\text {helical }}$ and different (negative and positive) values for the angle of inclination $\beta$. By definition, for a negative angle of inclination, the diameter of the laser beam path increases after passing the focal plane. For a large angle, this leads to a negatively conical borehole, i.e. the outlet in the
Table 1 Processing parameters

\begin{tabular}{ll}
\hline Parameter & Value \\
\hline Wavelength & $1030 \mathrm{~nm}$ \\
Pulse duration & $0.9 \mathrm{ps}$ \\
Repetition rate & $100 \mathrm{kHz}$ \\
Focal length & $100 \mathrm{~mm}$ \\
Focal diameter & $22 \mu \mathrm{m}$ \\
Defocusing length & $0 \mu \mathrm{m}$ \\
Pulse energy & $180 \mu \mathrm{J}$ \\
Helical diameter & $230 \mu \mathrm{m}, 208 \mu \mathrm{m}, 212 \mu \mathrm{m}$ \\
Angle of inclination & $-3.1^{\circ} ;-0.6^{\circ} ;+2.4^{\circ}$ \\
Rotation frequency & $27,988 \mathrm{~min}^{-1}$ \\
Polarization state & circular \\
Processing gas & Nitrogen $\left(\mathrm{N}_{2}, 2\right.$ bar $)$ \\
\hline
\end{tabular}

direction of propagation of the laser beam has a larger diameter than the inlet.

The drilling program was designed to produce the final borehole geometry in order to meet the assumptions of the model by keeping all the parameters constant for several tens of seconds.

For determining the exact values of the helical diameter $d_{\text {helical }}$ and the angle of inclination $\beta$, the following simple method was applied: For each parameter set of $d_{\text {helical }}$ and $\beta$, markings of the beam path have been produced in several defocusing planes on a flat sample. By rotating the laser beam with the helical drilling optics in a circular motion and shifting the focal plane from below to above the sample surface in discrete steps, rings of different diameter and line width can be marked with low pulse energy and short laseron-times.

As long as the beam spot does not overlap in one rotation, there is a clearly distinguishable inner and outer boundary of the rings. From their diameters, the mean diameter of each ring can be calculated, equaling the diameter of the circular movement of the laser beam's central axis. The angle of inclination $\beta$ and $d_{\text {helical }}$ can be computed from the pitch and intercept of a linearly regressed line of the course of the mean diameters with respect to the defocusing planes. The results of this investigation of $\beta$ and $d_{\text {helical }}$ can be found in Table 1.

The materials machined in the validation experiments are stainless steel (EN steel number 1.4301) and copper as metals, as well as undoped silicon and bulk aluminum oxide ceramic $\left(\mathrm{Al}_{2} \mathrm{O}_{3}\right.$, alumina) as non-metals. Flat samples have been used with a thickness of $1 \mathrm{~mm}$ (metals) and $0.65 \mathrm{~mm}$ (non-metals).

After drilling, all samples have been ground and polished to the central cross-sectional plane of the boreholes and recorded with a scanning electron microscope (SEM).

\section{Experimental and simulation results}

\subsection{Analysis of geometry}

In Fig. 1, SEM images of cross-sections of the boreholes produced in different materials using different drilling parameters are displayed. The laser entrance is on the top side, 

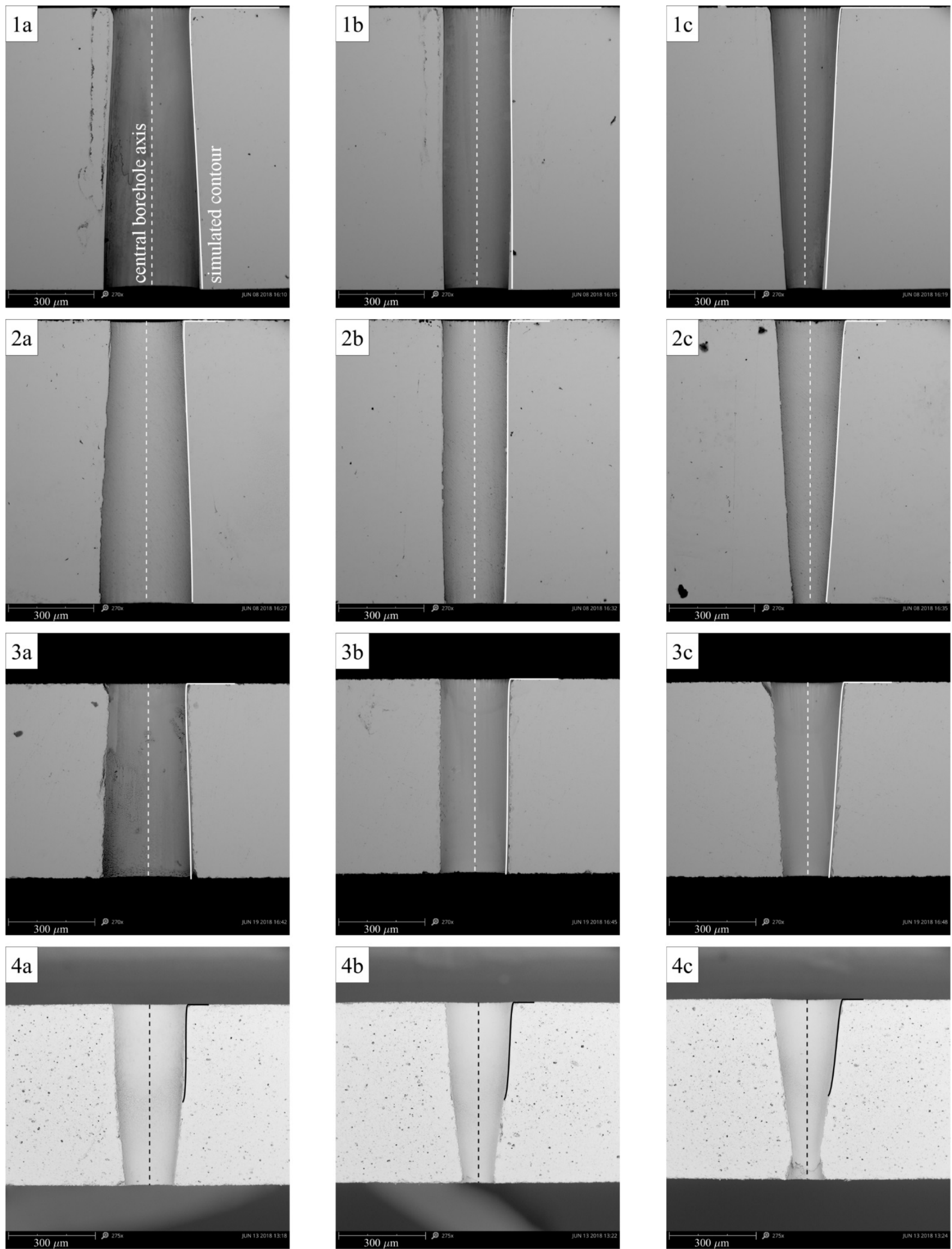

Fig. 1 SEM images of boreholes drilled into different materials with the respective simulated contour (white/black lines at right borehole edges), row 1: stainless steel, row 2: copper, row 3: undoped silicon, row 4: aluminum oxide ceramic, column a: negative angle of inclination $\beta=-3.1^{\circ}$, column $\mathrm{b}$ : negative angle of inclination $\beta=-0.6^{\circ}$, column c: positive angle of inclination $\beta=+2.4^{\circ}$. All other process parameters are given in Table 1. For the simulation, literature values for the material properties were used, see Table 2. 
the laser exit on the bottom side. At first, an analysis of the borehole geometries is presented.

For each material, three different boreholes are shown: drilled with a negative (first column, marked a), positive (third column, marked c) and slightly negative (middle column, marked b) angle of inclination. The exact values can be found in Table 1 .

In each row, boreholes drilled into the same material are displayed: in stainless steel (first row, marked 1), copper (second row, marked 2), undoped silicon (third row, marked 3) and alumina (fourth row, marked 4).

For all materials, the same drilling parameters were used, allowing a comparison of the boreholes.

Boreholes in stainless steel, copper, and silicon are quite similar in shape and size. Since the silicon samples have a smaller thickness, the boreholes are similar to the upper parts of the respective holes drilled into the metals. The drilling parameters result in negatively conical (a), cylindrical (b) and positively conical (c) forms. The entrance diameters for the different angles of inclination are almost equal in size (approx. $270 \mu \mathrm{m}$ ), whereas the exit diameters are varying strongly, leading to the different hole shapes (340 $\mu \mathrm{m}$ to 120 $\mu \mathrm{m}$ for the metals and $320 \mu \mathrm{m}$ to $170 \mu \mathrm{m}$ for silicon).

At the laser entrance side, a rounded corner from the top surface until approx. $40 \mu \mathrm{m}$ depth is visible. In contrast, at the laser exit side, there is a sharp edge to the bottom surface. The edge rounding at the laser entrance leads to the effect that the smallest diameter of the negatively conical boreholes is located a few tens of micrometers below the surface.

Comparing the materials discussed so far to the ceramic, huge differences in geometry are visible. No negatively conical or cylindrical hole could be produced, but all parameters were leading to positively conical shapes. The entrance diameters are a little bit smaller (approx. $260 \mu \mathrm{m}$ ), but the exits show very different characteristics: from a diameter of 170 $\mu \mathrm{m}$ (Fig. 1, 4a) there is a reduction to $120 \mu \mathrm{m}$ (Fig. 1, 4b) and an increase again to $140 \mu \mathrm{m}$ (Fig. 1, 4c).

Most noticeable in Fig. 1, 4c is the constriction (smallest diameter $95 \mu \mathrm{m}$ ) in the lower part of the hole and the subsequent opening.

\subsection{Simulation results}

For each borehole in Fig. 1, the corresponding simulated cross-sectional geometry is shown as a white/black line at the right edge of the hole. The borehole contour is assumed to be symmetrical and the central borehole axis is marked as a dashed line.

As described in Sec. 2, the two material parameters needed are the complex refractive index $n+i k$ and the absorbed fluence at the ablation threshold $\tilde{F}_{t h}$. Both parameters can be found in Table 2 for each material with values taken from literature sources.

It is most common to find values for the incident fluence at the ablation threshold $F_{\text {th,inc }}$ in literature. However, as can be seen from Eq. (1), the value of the absorbed fluence at the threshold $\tilde{F}_{t h}$ is required for the simulation. According to $\tilde{F}_{t h}=\left(1-R\left(0^{\circ}\right)\right) \cdot F_{t h, i n c}, \tilde{F}_{t h}$ can be computed from $F_{t h, i n c}$ with the help of the reflectivity for normal incidence, calculated from the Fresnel equations for circular polarization, as shown in Table 2.

Noticeable are the huge material-dependent variations in the reflectivity $R$ as well as in both incident and absorbed fluence at the ablation threshold with the absorbed fluence values spanning two orders of magnitude from copper to alumina.

Using these literature values for the material properties in the simulation, proves an overall good agreement between model and experiment. Especially values for the ablation threshold are subject to a variety of influences, including incubation behavior [14], wavelength [14] and pulse duration dependency [16]. Smaller differences between model and experiment, particularly at the exits of the steel samples and for silicon, might arise from this uncertainty in the ablation threshold or deviations of the beam profile from a Gaussian fluence distribution, especially far away from the focal plane.

For both metals and silicon, the simulated contours closely resemble the measured ones including all characteristics described in Sec. 4.1. So, the findings are supported by simulation.

From the simulation of the ceramic, an interesting effect becomes visible. The line of constant absorbed fluence which is assumed to equal the cross-sectional borehole geometry, ends halfway through the borehole, although the

Table 2 Material parameters for the simulation at $\lambda=1030 \mathrm{~nm}$ and $\tau=0.9 \mathrm{ps}$ : complex refractive index $\bar{n}=n+i k$, incident fluence at ablation threshold $F_{t h, i n c}$, reflectivity at normal incidence $\mathrm{R}\left(0^{\circ}\right)$, absorbed fluence at ablation threshold $\tilde{F}_{t h}$

\begin{tabular}{llllll}
\hline Material & $\mathrm{n}$ & $\mathrm{k}$ & $F_{\text {th,inc }} / \mathrm{J} \cdot \mathrm{cm}^{-2}$ & $\left(1-\mathrm{R}\left(0^{\circ}\right)\right)$ & $\tilde{F}_{t h} / \mathrm{J} \cdot \mathrm{cm}^{-2}$ \\
\hline Stainless steel & 2.59 & 4.87 & $7 \cdot 10^{-2}$ & 0.283 & $2.2 \cdot 10^{-2}$ \\
1.4301 & {$[10]$} & {$[10]$} & {$[14]$} & & \\
Copper & 0.34 & 6.77 & $3.3 \cdot 10^{-1}$ & 0.029 & $9.6 \cdot 10^{-3}$ \\
& {$[11]$} & {$[11]$} & {$[14]$} & & \\
Undoped & 3.57 & $2.4 \cdot 10^{-4}$ & $2.5 \cdot 10^{-1}$ & 0.684 & $1.7 \cdot 10^{-1}$ \\
silicon & {$[12]$} & {$[12]$} & {$[14]$} & & \\
Aluminum & $1.744^{1)}$ & $0.021)$ & $1.1 \cdot 10^{02}$ & 0.927 & $1.02 \cdot 10^{0}$ \\
oxide ceramic & {$[13]$} & {$[13]$} & {$[15]$} & & \\
\hline
\end{tabular}

1) Arithmetic mean of both values for parallel and perpendicular polarization

2) Value was actually determined for $\lambda=775 \mathrm{~nm}$ and $\tau=0.18 \mathrm{ps}$ 
sample thickness amounts to only $65 \%$ of the metallic samples. This means that from this point on, the absorbed fluence at the hole surface falls below the ablation threshold fluence everywhere.

A possible explanation for the discrepancy of modelled and measured borehole geometries might be a change of the material parameters with increasing hole depth as a reaction to the laser treatment. As constant material properties are assumed in the model, this behavior cannot be simulated.

Another possibility comes from the assumptions of the borehole model [7]: Only direct absorption of the laser beam is considered, reflected rays are neglected.

Apparently, however, reflected radiation is reabsorbed at the hole walls, especially in the bottom half. The combination of reabsorbed reflected radiation and the low directly absorbed fluence must exceed the ablation threshold to ablate material further than predicted by the model.

The deeper and narrower a borehole gets, the higher is the amount of reabsorbed reflected radiation in comparison to directly absorbed light. For the ceramic this gets significant, as the directly absorbed radiation is not sufficient to exceed the ablation threshold in the lower part of the hole. Presumably, the reflected radiation is mostly accountable for the hole geometry in the bottom half, including the characteristic of the constriction in Fig. 1, 4c.

In fact, in the top half, the slope of the borehole contour is changing according to the angle of inclination (comparing Fig. 1, 4a-4c) which is well reproduced by the simulated geometry. This means that there is a negligible amount of reabsorbed reflected radiation in the top half of the boreholes. Furthermore, there seems to be a "knee" at half depth of the holes in Fig. 1, 4a,b, possibly marking the change between the two regimes described.

For the other materials a similar behavior might be observable for deeper boreholes, as the maximum point of the calculated line of constant absorbed fluence is below the bottom surfaces of the samples used.

\section{Conclusion}

For helical drilling using ultrashort laser pulses, a model for the final borehole geometry was presented. This model is based on calculating a line of constant absorbed fluence defined by the ablation threshold fluence. For validating the model for different materials, boreholes have been drilled into stainless steel, copper, undoped silicon, and aluminum oxide ceramic with drilling parameters resulting in holes of different shape and size. While the geometries of both metals and silicon were similar, they differed characteristically for the ceramic, although the same drilling parameters had been applied.

For the simulation, several parameters have been determined experimentally, including the exact angle of inclination and helical diameter. Material properties have been taken from literature sources. The simulated borehole geometries are in good agreement with all experimental results. For the ceramic, however, the simulated contour stops halfway through the hole. Different reasons have been proposed for this behavior. Still, the top half is in good agreement with the model. For a simulation of the complete geometry, further research is needed.

\section{References}

[1] C. Föhl, D. Breitling, K. Jasper, J. Radtke and F. Dausinger: Proc. SPIE 4426 - Second International Symposium on Laser Precision Microfabrication, (2002) 104.

[2] R. Giedl, H.-J. Helml, F.X. Wagner and M.J. Wild: Proc. SPIE 5063 - Fourth International Symposium on Laser Precision Microfabrication, (2003) 389.

[3] L. Romoli, C.A.A. Rashed and M. Fiaschi: Optics Laser Technol., 56, (2014) 35.

[4] D. Jahns, T. Kaszemeikat, N. Mueller, D. Ashkenasi, R. Dietrich and H.J. Eichler: Phys. Proc., 41, (2013) 630.

[5] R. Kling, M. Dijoux, L. Romoli, F. Tantussi, J. Sanabria and E. Mottay: Proc. SPIE 8608 - Laser-based Microand Nanopackaging and Assembly VII, (2013) 86080F

[6] C. Fornaroli, J. Holtkamp and A. Gillner, Phys. Proc., 41, (2013) 661.

[7] A. Kroschel, A. Michalowski and T. Graf: Adv. Opt. Techn., 7, (2018) 183.

[8] S. Döring, J. Szilagyi, S. Richter, F. Zimmermann, M. Richardson, A. Tünnermann and S. Nolte: Opt. Express, 20, (2012) 27147.

[9] J.M. Liu: Opt. Lett. 7, (1982) 196.

[10]A. Michalowski: "Untersuchungen zur Mikrobearbeitung von Stahl mit ultrakurzen Laserpulsen“, PhD Thesis (Herbert Utz Verlag, Munich, 2014) p. 51. (in German)

[11] P.B. Johnson and R.W. Christy: Phys. Rev. B, 6, (1972) 4370.

[12] M.R. Ruben: "Development of physical models for the simulation of optical properties of solar cell modules", PhD Thesis (Hannover, 2015) p. 141.

[13] M.R. Querry: “Optical constants”, PhD Thesis (Kansas City, Missouri, 1985) pp. 38, 55.

[14]B. Neuenschwander, B. Jaeggi, M. Schmid, V. Rouffiange and P.-E. Martin: Proc. SPIE 8243 - Laser Applications in Microelectronic and Optoelectronic Manufacturing, (2012) 824307.

[15] W. Perrie, A. Rushton, M. Gill, P. Fox and W. O'Neill: Proc. SPIE 5714 - Commercial and Biomedical Applications of Ultrafast Lasers V, (2005) 43.

[16] N. Lasemi, U. Pacher, L.V. Zhigilei, O. Bomatí-Miguel, R. Lahoz and W. Kautek: Appl. Surf. Sci., 433, (2018) 772 .

(Received: June 24, 2018, Accepted: November 11, 2018) 